Neurosurg Focus 18 (1):E7, 2005

\title{
Carotid artery angioplasty and stent placement for recurrent stenosis
}

\author{
Christopher J. Koebbe, M.D., Kenneth Liebman, M.D., Erol Veznedaroglu, M.D., \\ AND ROBERT ROSENWASSER, M.D.
}

Department of Neurological Surgery, Thomas Jefferson University School of Medicine, Philadelphia, Pennsylvania

\begin{abstract}
Object. The use of endovascular management for recurrent carotid artery (CA) stenosis is rapidly expanding due to the increased surgical risk associated with repeated carotid endarterectomy (CEA). Carotid artery angioplasty and stent placement for recurrent CA stenosis offers a less invasive strategy with fewer procedural complications and may provide a more durable treatment. The authors report on their experience with this procedure in the management of recurrent CA stenosis.

Methods. A retrospective review was performed to evaluate clinical and ultrasound imaging outcomes after CA angioplasty and stent placement. Twenty-three vessels in 22 patients with severe recurrent stenosis $(>80 \%)$ post-CEA were treated with balloon angioplasty and stent placement without distal protection. There were no perioperative neurological or cardiac complications in this series. Over a mean follow-up period of 36 months, one patient (5\%) suffered recurrent stenosis requiring retreatment with angioplasty alone.

Conclusions. The use of CA angioplasty and stent placement provides a safe and effective treatment for recurrent CA stenosis. The use of drug-eluting and/or bioactive stents in the future will likely further improve the efficacy of this procedure for recurrent CA stenosis.
\end{abstract}

KEY WORDS - carotid artery stenosis

- angioplasty - carotid stent placement

Carotid endarterectomy has been proven to be more effective in preventing stroke when compared with medical management in patients with severe symptomatic and asymptomatic atherosclerotic CA stenosis. ${ }^{2,9}$ In the North American Symptomatic Carotid Endarterectomy Trial and the Asymptomatic Carotid Atherosclerosis Study it was determined that the rates of morbidity and mortality should be less than 6 and 3\%, respectively, to realize the benefit of surgery over medical management. Given the higher perioperative risk factors of CEA for recurrent stenosis, the balloon angioplasty and stent placement procedure has been advocated. ${ }^{1,10}$ For this recommendation to be acceptable, the morbidity and mortality rates of stentassisted angioplasty in the CA must be below those for CEA. As illustrated in the Stenting and Angioplasty with Protection in Patients at High Risk for Endarterectomy trial, high-risk surgical candidates have a lower procedure-related morbidity after CA angioplasty and stent placement than occurs following CEA. ${ }^{15}$ The purpose of this study was to analyze retrospectively our experience with CA angioplasty and stent placement in recurrent stenosis after CEA.

Abbreviations used in this paper: $\mathrm{CA}=$ carotid artery; $\mathrm{CCA}=$ common $\mathrm{CA}$; CEA = carotid endarterectomy.

\section{CLINICAL MATERIAL AND METHODS}

A retrospective review was performed to evaluate clinical and Doppler imaging follow up in patients who underwent CA angioplasty and stent placement for recurrent stenosis after CEA. All procedures were performed in the interventional neuroradiology suite by using biplane digital fluoroscopy. The procedures were performed after induction of general anesthesia and with somatosensory evoked potential monitoring. All patients were pretreated with aspirin and clopidogrel for at least 3 days before the procedure. Because of the risk of bradycardia or asystole, the Zoll transcutaneous pacer was used in all patients. This device paced the HR in approximately $30 \%$ of these patients, similar to the frequency among first-time CA angioplasty and stent placement procedures, with an increased incidence of firing when the lesion was close to the carotid bulb/sinus.

Initially, a No. 5 French femoral sheath (Cordis Endovascular, Johnson and Johnson Corp., Miami, FL) was placed, and arteriography was performed through a No. 5 French Berenstein diagnostic catheter (Cordis Endovascular, Johnson and Johnson Corp.). Measurements were made of the degree and length of stenosis as well as the diameter of the internal carotid artery above and the CCA below the stenosis (Fig. 1a). These measurements were 


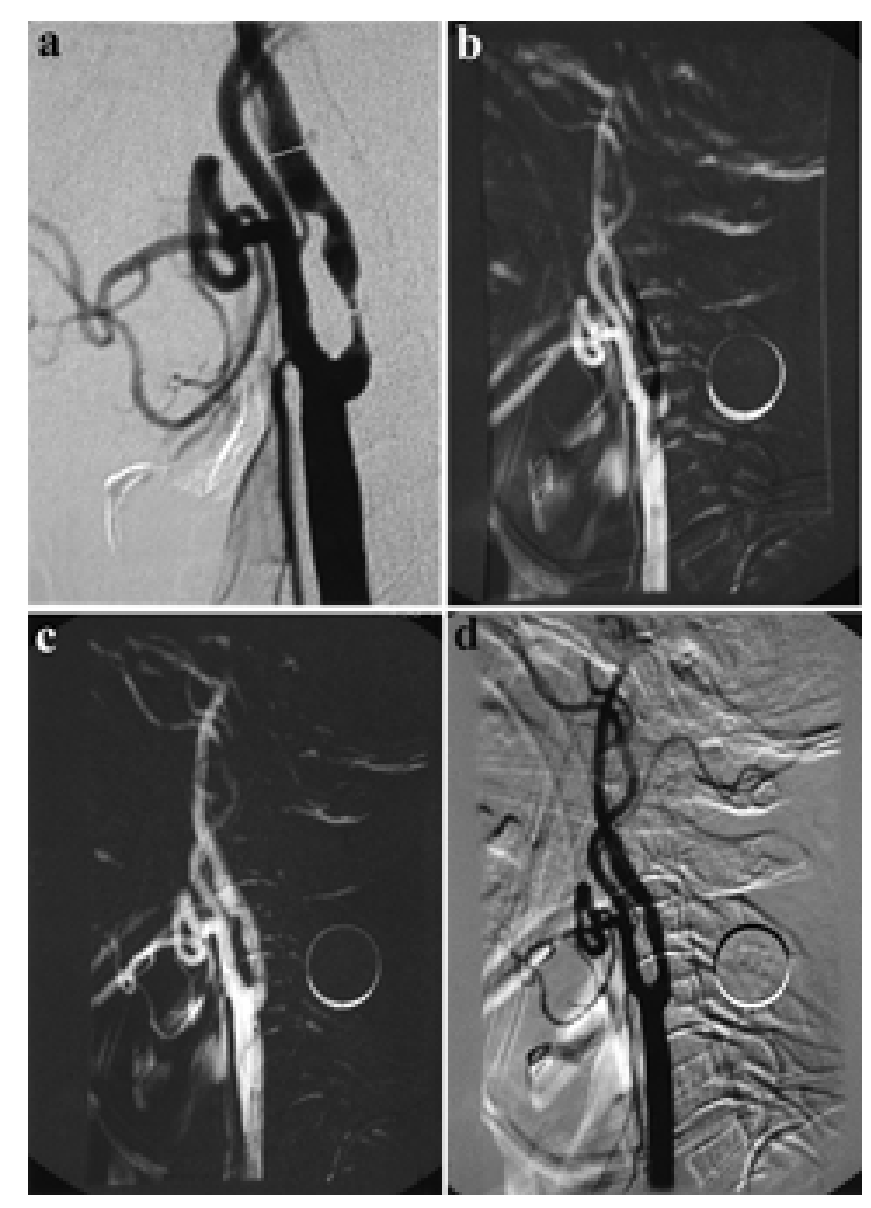

Fig. 1. a: Lateral view of a CCA injection demonstrating $90 \%$ stenosis of the internal carotid artery 12 months after CEA. b: Digital subtraction roadmap angiography demonstrating initial balloon inflation. c: Postangioplasty roadmap view obtained before stent delivery, demonstrating improvement in vessel diameter. d: Final lateral view of the CA bifurcation after stent placement, demonstrating significant resolution of recurrent stenosis.

important to determine the appropriate stent diameter and length. The femoral sheath was then converted to a No. 8 French sheath to accommodate the angioplasty balloon and stent. A No. 8 French $90-\mathrm{cm}$ guiding catheter (Vista Brite Tip; Cordis Endovascular, Johnson and Johnson Corp.) was positioned in the CCA. Based on their weight, patients then received boluses of heparin and were maintained on a continuous drip to keep the activated clotting time at two times the baseline value.

With the aid of roadmap guidance, a 0.35 to 0.38 -in Terumo guidewire (Boston Scientific, Fremont, CA) was passed through the stenosis. The Savvy angioplasty balloon catheter system (Cordis Endovascular, Johnson and Johnson Corp.) was then passed over the wire, across the stenosis, and the balloon was inflated up to 8 to $10 \mathrm{~atm}$ (Fig. 1b). The pressure of balloon inflation was dependent on tactile and radiographic assessment. The number of balloon inflations was determined based on pre- and postdilation angiograms. A follow-up angiogram was obtained after each inflation. If results of the angiogram were not satisfactory, repeated angioplasty was performed (Fig. 1c). Once angioplasty was deemed adequate on the pre- liminary angiogram, a nitinol transhepatic biliary system stent and catheter system was passed across the diseased area under roadmap guidance.

The types of stents used in our series included 22 SMART (Cordis Endovascular) and one ACCULINK (Guidant Corp., Santa Clara, CA) device. The size of stents used in our series varied in length from 20 to $40 \mathrm{~mm}$ and in diameter from 5 to $8 \mathrm{~mm}$. After the device was positioned, a follow-up angiogram was obtained to ensure that the postdelivery dilation of the stent was adequate (Fig. 1d). If not, balloon dilation was performed after delivery of the stent. Once treatment was completed, a lowmagnification angiogram was obtained to view the carotid bifurcation and intracranial vessels to evaluate for thromboembolic events. Postoperatively, patients were maintained on a continuous heparin infusion for 24 to 48 hours to a goal activated clotting time of 2 to 2.5 times baseline. Patients were discharged on a maintenance dose of aspirin and clopidogrel.

\section{RESULTS}

Twenty-three vessels in 22 patients were treated using CA angioplasty and stent placement for recurrent stenosis after CEA. The mean patient age was 71 years. The mean period between CEA and CA angioplasty and stent placement was 28 months. All patients had high-grade stenosis $(>80 \%)$ on angiography. Twenty-two patients presented with transient ischemic attacks or amaurosis fugax, whereas one was asymptomatic. There were no neurological or cardiac complications in this series in the perioperative period. In one patient a groin/retroperitoneal hematoma developed, which required blood transfusion and delayed hospital discharge. During a mean clinical follow-up period of 36 months no further ischemic events occurred in this series of patients. Follow-up duplex ultrasonography studies performed every 6 months after CA angioplasty and stent placement were available on 20 vessels in 19 patients and demonstrated one case of recurrent stenosis (4\%) 14 months after CA angioplasty and stent placement, which was treated with angioplasty alone.

\section{DISCUSSION}

The incidence of recurrent stenosis after CEA ranges from 2 to $50 \%$, depending on the routine use of follow-up imaging studies, follow-up duration, and methods/criteria for defining restenosis. Two distinct pathophysiological processes are responsible for recurrent CA stenosis. Stoney and String ${ }^{11}$ found that early restenosis $(<24$ months postoperatively) was due to fibrous myointimal hyperplasia, whereas late restenosis ( $\geq 24$ months) was due to progressive atherosclerotic disease of the vessel. Surgical management of recurrent CA stenosis is technically more challenging, leading to increased complication rates compared with the initial endarterectomy. Meyer, et al., ${ }^{7}$ described an $11 \%$ major morbidity/mortality rate with repeated CEA due to the difficulty of dissecting scarred planes between the recurrent atheroma and underlying media and due to the friable nature of a recurrent plaque, increasing the risk of emboli. The durability of reoperation for recurrent disease is also an issue; delayed failure, including ischemic events, is as high as $20 \% .^{3}$ The need 
for a safer and more durable treatment prompted the application of endovascular techniques to manage recurrent CA disease.

Tievsky, et al., ${ }^{13}$ published one of the first reports of balloon angioplasty for the management of postoperative restenosis following CEA in 1983. They described successful treatment of a CCA plaque attributed to clamp injury during a second CEA in a patient with severe coronary disease status after cardiac bypass with ventricular arrhythmias. In another early series, investigators described successful revascularization in four of five vessels treated with balloon angioplasty alone without neurological complications. ${ }^{12}$ The initial experience with CA angioplasty and stent placement for recurrent stenosis demonstrated a stroke rate of $4 \%$ and no evidence of restenosis on 6-month postprocedure angiography. ${ }^{14}$ The addition of stents to angioplasty for the endovascular management of recurrent CA stenosis reduces the risk of emboli from a friable vessel wall and provides a more durable endoluminal frame. Lanzino, et al., ${ }^{5}$ evaluated outcomes of angioplasty alone and CA angioplasty and stent placement, demonstrating a higher recurrence rate in the group treated with angioplasty alone, with no neurological complications in 16 patients over a mean follow-up duration of 27 months. In a number of single-institution studies, the CA angioplasty and stent placement procedure was compared with repeated CEA for recurrent CA disease, and angioplasty and stent placement was found to be equivalent or better in terms of neurological outcome and restenosis rates between the two treatment groups..$^{1,4,10}$

We successfully treated 23 vessels in 22 patients with no neurological complications and one case of restenosis over a mean follow-up duration of 36 months. There was one groin complication (4.5\%) in this series, which is slightly higher than our overall experience with CA angioplasty and stent placement, for which the incidence is $2 \%$. This compares favorably with the 4 to $10 \%$ risk of wound hematoma or infection and cranial nerve injury associated with repeated endarterectomy. ${ }^{3,6}$ Although no thromboembolic complications occurred in our series, endovascular management of CA stenosis after CEA allows for recognition and treatment with intraarterial mechanical devices and/or thrombolytic agents during the procedure. The role of distal protection devices for CA angioplasty and stent placement for recurrent CA disease has not been well examined, and in our series there were no neurological complications when distal protection was not used.

It is important to note that there were no cardiac events, despite severe coronary artery disease in most patients in our series. For patients at high risk of cardiac or other medical complications during repeated CEA, CA angioplasty and stent placement requires less anesthesia time and can be performed with patients in a state of conscious sedation, thus reducing complications attributed to general anesthesia. The main issue in comparing repeated surgery with CA angioplasty and stent placement for recurrent $\mathrm{CA}$ stenosis is durability. The introduction of drug-eluting stents has revolutionized the management of coronary artery disease by significantly reducing the incidence of restenosis. ${ }^{8}$ The application of these devices to CA angioplasty and stent placement will likely improve long-term outcomes, providing an even greater advantage for endovascular treatment of recurrent CA stenosis. There is an ongoing need to compare CA angioplasty and stent placement to CEA in a prospective randomized fashion. Many ongoing trials should provide a larger patient experience and more definitive outcomes on which management decisions for CA disease will be based.

\section{CONCLUSIONS}

Our experience with CA angioplasty and stent placement in a limited number of patients confirms the safety and durability of endovascular management of recurrent CA stenosis. The application of bioactive and drug-eluting stents in the future should further improve the durability of endovascular management of recurrent stenosis after CEA.

\section{References}

1. Bowser AN, Bandyk DF, Evans A, et al: Outcome of carotid stent-assisted angioplasty versus open surgical repair of recurrent carotid stenosis. J Vasc Surg 38:432-438, 2003

2. Executive Committee for the Asymptomatic Carotid Atherosclerosis Study. Endarterectomy for asymptomatic carotid artery stenosis. JAMA 273:1421-1428, 1995

3. Gagne PJ, Riles TS, Jacobowitz GR, et al: Long-term follow-up of patients undergoing reoperation for recurrent carotid artery disease. J Vasc Surg 18:991-1001, 1993

4. Hobson RW II, Goldstein JE, Jamil Z, et al: Carotid restenosis: operative and endovascular management. J Vasc Surg 29: 228-238, 1999

5. Lanzino G, Mericle RA, Lopes DK, et al: Percutaneous transluminal angioplasty and stent placement for recurrent carotid artery stenosis. J Neurosurg 90:688-694, 1999

6. Mansour MA, Kang SS, Baker WH, et al: Carotid endarterectomy for recurrent stenosis. J Vasc Surg 25:877-883, 1997

7. Meyer FB, Piepgras DG, Fode NC: Surgical treatment of recurrent carotid artery stenosis. J Neurosurg 80:781-787, 1994

8. Moses JW, Leon MB, Popma JJ, et al: Sirolimus-eluting stents versus standard stents in patients with stenosis in a native coronary artery. N Engl J Med 349:1315-1323, 2003

9. North American Symptomatic Carotid Endarterectomy Trial Collaborators. Beneficial effect of carotid endarterectomy in symptomatic patients with high-grade carotid stenosis. N Engl J Med 325:445-453, 1991

10. Rockman CB, Bajakian D, Jacobowitz GR, et al: Impact of carotid artery angioplasty and stenting on management of recurrent carotid artery stenosis. Ann Vasc Surg 18:151-157, 2004

11. Stoney RJ, String ST: Recurrent carotid stenosis. Surgery 80: 705-710, 1976

12. Theron J, Raymond J, Casasco A, et al: Percutaneous angioplasty of atherosclerotic and postsurgical stenosis of carotid arteries. AJNR 8:495-500, 1987

13. Tievsky AL, Druy EM, Mardiat JG: Transluminal angioplasty in postsurgical stenosis of the extracranial carotid artery. AJNR 4:800-802, 1983

14. Yadav JS, Roubin GS, King P, et al: Angioplasty and stenting for resatenosis after carotid endarterectomy. Initial experience. Stroke 27:2075-2079, 1996

15. Yadav JS, Wholey MH, Kuntz RE, et al: Protected carotidartery stenting versus endarterectomy in high-risk patients. $\mathbf{N}$ Engl J Med 351:1493-1501, 2004

Manuscript received November 30, 2004.

Accepted in final form December 20, 2004.

Address reprint requests to: Christopher J. Koebbe, M.D., Department of Neurological Surgery, Thomas Jefferson University, 909 Walnut Street, 3rd Floor, Philadelphia, Pennsylvania 19107. email: chriskoebbe@hotmail.com. 